\title{
Study on the Short Term Prediction of Satellite Cloud Image Based on Curve Fitting
}

\author{
Huang Min ${ }^{1}$ and ${ }^{*}$ Wang Jiali $^{2}$ \\ ${ }^{1,2}$ School of Computer and Communication Engineering, Zhengzhou University of \\ Light Industry \\ ${ }^{1}$ Huangmin1998@126.com \\ jiali1218@foxmail.com
}

\begin{abstract}
The satellite cloud images can display the change of weather system. The precise prediction of cloud can improve the accuracy of weather forecasts. So we propose the idea of curve fitting for short term prediction of cloud cluster movement in the satellite cloud image. According to the previous moving track of cloud, we can work out the result by the curve fitting. Then our proposed algorithm use clouds motion vector to predictive emendation. Experimental results show that the method has the intuitive, quantitative and accurate advantages for relatively stable cloud cluster movement of short-term forecasting in the satellite cloud image.
\end{abstract}

Keyword: satellite cloud image; SSDA; curve fitting; motion vector

\section{Introduction}

Satellite cloud image is a picture above the earth, which is covered by clouds cluster and the earth's surface. The satellite cloud images have rich meteorological information. The use of satellite cloud images can identify different weather systems, determine their location, and estimate the intensity. If we know the cloud cluster movement trajectory, we can conjecture the trend of weather development and the moving direction. So we can use satellite cloud images to provide a basis for the analysis and prediction of meteorological disasters. In order to make more use of satellite cloud image, we need to study the method of predicting cloud cluster movement trajectory.

In recent years, cloud cluster movement trajectory prediction based on various methods obtained fast development. Wolf D E[1] put forward can process satellite data to obtain cloud motion vectors for certain research studies, or for input to numerical prediction models; Xuan $\mathrm{L}[2]$ proposed a novel efficient algorithm for motion detection in dynamic background; Huang Y[3] was used the maximum correlation and linear extrapolation to obtain a new method for the prediction of cloud cluster; Liu K F[3] introduced the idea of cross correlation in cloud cluster movement of short-term prediction. In the next two years, Liu K $\mathrm{F}[4]$ was proposed another method by using singular value decomposition combined with radial basis function neural network; Wang J G[5] etc. was proposed a cloud nonlinear prediction model inversion method based on the idea of empirical orthogonal function (EOF) and genetic algorithm parameters optimization. However, these algorithms have shortcoming, such as large amount of computations and slow computing speed. In order to solve these problems, we introduce the curve fitting in the cloud forecast. Combined with the research results of predecessors, our proposed algorithm uses the motion vector to correct the prediction results. This can increase the accuracy of prediction. We put forward a new point of view to solve the problem of cloud prediction. This method has the advantages of clear logic, simple operation and accurate prediction.

In this paper, we use the satellite images which from FY2H. According to the previous 
moving track of cloud, we can work out the result by the curve fitting. Then our proposed algorithm use clouds motion vector to predictive emendation. Experimental results show that the method has the good feasibility.

\section{Related Theory}

\subsection{Sequential Similarity Detection Algorithm}

If there are image $\mathrm{S}$ and template image $\mathrm{T}$, and $\mathrm{S}$ ' size is $\times N$, and T' size is $M \times M$. How to find the specific location of the template image $T$ in the image $S$ ? The most direct approach is to put the template image $\mathrm{T}$ directly stacked on the image $\mathrm{S}$, than translation and comparison between the template image $\mathrm{T}$ and the sub graph $S^{i, j} .(i, j)$ is a sub graph in the upper left corner of the image coordinates. This method is the traditional template matching ${ }^{[6]}$.

Sequential similarity detection algorithm (SSDA) ${ }^{[7]}$ is an improvement of the traditional template matching algorithm, and the speed of the algorithm is improved by reducing the number of pixels and the number of similar comparisons. The algorithm flow is to calculate the error value e between sub graph and template image corresponding points, and its accumulation summation $\mathrm{E}$, if $\mathrm{E}$ is less than the threshold $\mathrm{T}^{\prime}$, and continue to select the pixel points in the sub graph to calculate the error value and the sum; if $\mathrm{E}$ is greater than the threshold T', move the template image to the next position, until the end of the image. Select the maximum number of cumulative times $\mathrm{C}$ position, that is, the matching position.

The error value e is:

$$
e\left(i, j, m_{k}, n_{k}\right)=\left|S^{i, j}\left(m_{k}, n_{k}\right)-\widehat{S^{\imath, j}}-T(m, n)+\widehat{T}\right|
$$

$\widehat{S^{i, j}}$ denotes the pixel average of the sub graph $S^{i, j}$ under the template image T, $\widehat{T}$ signals the average value of the template pixel.

\subsection{Curve Fitting Algorithm}

If there is a function of the numerical table, as shown in Table 1, it is now looking for a formula that can be approximately represented by these data. The problem is actually the approximation of the discrete function, and the curve fitting can solve the problem well. In this paper, we use the least square method of curve fitting [8].

\section{Table 1. Data Table}

\begin{tabular}{|c|c|c|c|c|c|}
\hline$x_{i}$ & $x_{1}$ & $x_{2}$ & $x_{3}$ & $\ldots$ & $x_{m}$ \\
\hline$f\left(x_{i}\right)$ & $f\left(x_{1}\right)$ & $f\left(x_{2}\right)$ & $f\left(x_{3}\right)$ & $\ldots$ & $f\left(x_{m}\right)$ \\
\hline
\end{tabular}

The function table in the function space $\phi$, seek $s^{*}(x)$, and

$$
\sum_{i=0}^{m} \omega_{i}\left[s^{*}\left(x_{i}\right)-f\left(x_{i}\right)\right]^{2}=\min _{s(x) \epsilon \phi} \sum_{i=0}^{m} \omega_{i}\left[s\left(x_{i}\right)-f\left(x_{i}\right)\right]^{2}
$$

This is the least squares problem of curve fitting, where the $\omega_{i}$ is described the right of the point $x_{i}$. The essence of the problem is that the $f(x)$ is the best approximation problem for the discrete function. The least square method of curve fitting can be used for the optimal square approximation of continuous functions. Specific practices are as follows:

Seek $s^{*}(x)$ is equivalent to solving multivariate functions' minimal value.

$$
I\left(a_{0}, a_{1}, \ldots, a_{n}\right)=\sum_{i=0}^{m} \omega_{i}\left(\sum_{j=0}^{n} a_{j} \varphi_{j}\left(x_{i}\right)-f\left(x_{i}\right)\right)^{2}
$$

The necessary conditions for the extreme values of the equation can obtain normal equation 
$\sum_{j=0}^{n}\left(\varphi_{j}, \varphi_{k}\right) a_{j}=\left(f, \varphi_{k}\right), k=0,1, \ldots, n$

inner product express addition type, that is

$\left\{\left(\varphi_{j}, \varphi_{k}\right)=\sum_{i=0}^{m} \omega_{i} \varphi_{j}\left(x_{i}\right) \varphi_{k}\left(x_{i}\right)\right.$

$\left\{\left(f, \varphi_{k}\right)=\sum_{i=0}^{m} \omega_{i} f\left(x_{i}\right) \varphi_{k}\left(x_{i}\right)\right.$

Because $\varphi_{0}(x), \varphi_{1}(x), \ldots, \varphi_{n}(x)$ are linear independence, the coefficient matrix of the normal equation (4) can be proved nonsingular. So we can solve this equation to obtain $a_{k}=a_{k}^{*}, k=0,1, \ldots n$, as to get $s^{*}(x)$. It's there and the only one.Call $s^{*}(x)$ is $f(x)^{\prime}$ ' least square approximation function in the function space $\phi$.

\subsection{Motion Vector}

The ultimate goal of the motion estimation is to find the motion vector and the prediction error by using the spatial redundancy of the video image in the image compression encoding [9]. In this paper, we use the motion vector to correct the prediction value.

Design $\mathrm{t}$ time of satellite cloud images as the current image $f(x, y), \mathrm{t}$ ' time of satellite cloud images as the reference image $f^{\prime}(x, y)$, the cloud clusters deviation between the current image and the reference image is the motion vector, it is a 2 dimensional vector ${ }^{[10]}$. It provides the cloud clusters' offset distance from the current image to the reference image.

\section{Proposed Algorithm Model}

This part describes the specific algorithm design. This algorithm specifically includes four major steps, shown in Figure 1, the process is as follows: image preprocessing, cloud matching, cloud prediction and cloud correction. The following is the details.

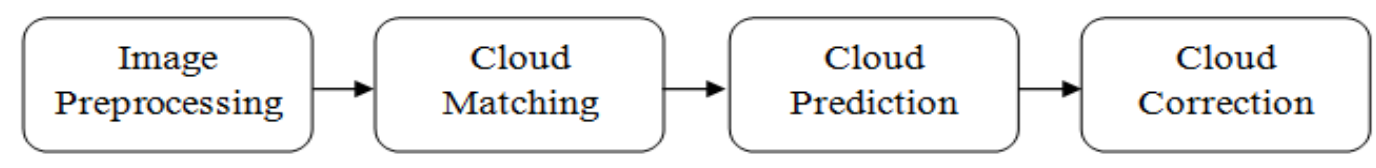

Figure 1. Flowchart of Design

\subsection{Image Preprocessing}

The infrared cloud images are selected as the test area of cloud image prediction, which from the first generation of geostationary orbit meteorological satellite FY-2. The satellite cloud images have more or less interference and noise, and these interference and noise can be reduced the cloud image quality, and they will have a certain impact on the forecast. In order to reduce the influence of the interference and noise on the image prediction, we first need to use smooth filter to eliminate the interference and noise in the cloud images.

In order to predict the moving trajectory of target cloud cluster by using curve fitting method, a unified working space coordinate system must be defined. The origin of this research is to define the upper left corner of the image of the same size and the same area. So it must be careful to select the same time interval, the same size of the satellite cloud image.

Prediction of cloud moving trajectory is mainly applied to rainfall or typhoon forecast, so relatively slow motion of clear sky clouds and meteorological cloud can assume that it does not exist. We only need to study the target cloud, which helps to speed up the matching to get accurate cloud. 


\subsection{Cloud Matching}

Cloud clusters in the sky are changing, moving, overlapping, disappearing constantly, so the cloud clusters in the satellite cloud image are mostly irregular. The matching of cloud images is a non precision matching problem, so we can use sequence similarity detection algorithm, it can exhibit good performance.

The core idea of sequence similarity detection algorithm: we need find the sub graph that is nearest and most closely related the template image $\mathrm{T}$ in all sub graph $S^{i, j}$. We do not care about the exact error values between other sub graph and template images in the process of searching the ultimate sub graph. What we can do is, according to the actual application environment select a proper threshold T', make the error accumulation summation between the matched sub graph and the template image less than or equal to the threshold value. In the calculation of the error between the sub image and the template image, if the error of the corresponding point is not calculated, the accumulated error has exceeded the threshold value, then it can be judged that the sub image is not a template image of the matched image, then stop calculation process of the error and accumulated summation, template image moved to the next position. Here, the number of pixels and the similarity of the search are reduced, and the amount of computation is reduced.

Sequential similarity detection algorithm is a search in the full image, and the range of the image is relatively large, although in image pre processing stage are filtered out part of the cloud, the algorithm can reduce the large amount of computation, but it still cannot meet the requirement of calculation speed. The cloud prediction is a short-term forecast, the shooting time between adjacent images is only an hour, and the moving range of cloud clusters within an hour is not too large on the satellite cloud image, which makes it possible to search for the small area of the image. We can search the neighborhood of the sub graph to reduce the search range. Practice in the field of $32 \times 32$ pixel search. This greatly improves the search speed, but also ensures the accuracy of the search.

\subsection{Cloud Prediction}

In order to use curve fitting method to predict the cloud, it is first necessary to screen a series of data, these data can represent the relative position of the cloud movement. We have already passed the selection of the same size, the same background of the same cloud to achieve the requirements of the unified coordinate space.

In this paper, we use the maximum inner rectangle to represent the relative position of the cloud. This is because that the clouds are in subtle changes all the time, so the maximum inner rectangle contains not only the cloud cluster's position change but also the cloud cluster's contour changes. If the maximum inner rectangle is selected, the maximum inner rectangle contains more information. So selection of the maximum inner rectangle has a good effect.

We use the four vertices of the maximum inner rectangle to predict the cloud cluster trajectory. For the cloud cluster trajectory prediction, we need to use the method of curve fitting for these four vertices, respectively. In order to use the method of curve fitting for every vertice, we need to decomposition of a vector in the horizontal and vertical direction. During cloud cluster trajectory prediction processing, we use curve fitting respectively in horizontal and vertical direction, and establish curve fitting equation of every vertice in horizontal and vertical direction with relative time change. Prediction equation form as shown in equation (6), $\mathrm{n}$ is the maximum number of equations. In the horizontal and vertical directions, the prediction equation is based on the need to select the appropriate number of times. $p_{n}, p_{n-1}, \ldots, p_{1}, p_{0}$ is constant term.

$$
x(t)=p_{n} x^{n}+p_{n-1} x^{n-1}+\cdots+p_{1} x+p_{0}
$$

Using the fitting results to predict the horizontal and vertical directions respectively, and then carry out the vector synthesis to get the preliminary results of the prediction. 


\subsection{Cloud Correction}

This paper uses the average value of cloud motion vector to obtain an amendment image. The $\mathrm{N}$ continuous times satellite cloud image have same interval time, respectively marked as $\mathrm{t}_{0}-(\mathrm{N}-1), \mathrm{t}_{0}-(\mathrm{N}-2), \ldots \mathrm{t}_{0}-1, \mathrm{t}_{0}$. Two adjacent cloud images, the first picture is the reference image $f^{\prime}(x, y)$, Second pictures is the current image $f(x, y)$, then we can compute the motion vector between each adjacent cloud images. The motion vector provides the offset between reference image and the current image. The average value of $\mathrm{N}-1$ motion vector is calculated. The cloud cluster in the current image goes on some offset in this direction of average motion vector to can get the correct image.

We select the intermediate position between the cloud position of the correction image and the cloud position of the forecasted image as the final result. This is the correction results in this paper.

\section{Experimental Results Analysis}

From the Central Meteorological Observatory, we can obtain the infrared cloud images of consecutive times of the October 2015 by the FY-2 weather satellite. In the area of $380 \times 385$ pixels in the cloud image, using the above calculation scheme for the cloud cluster prediction experiment.

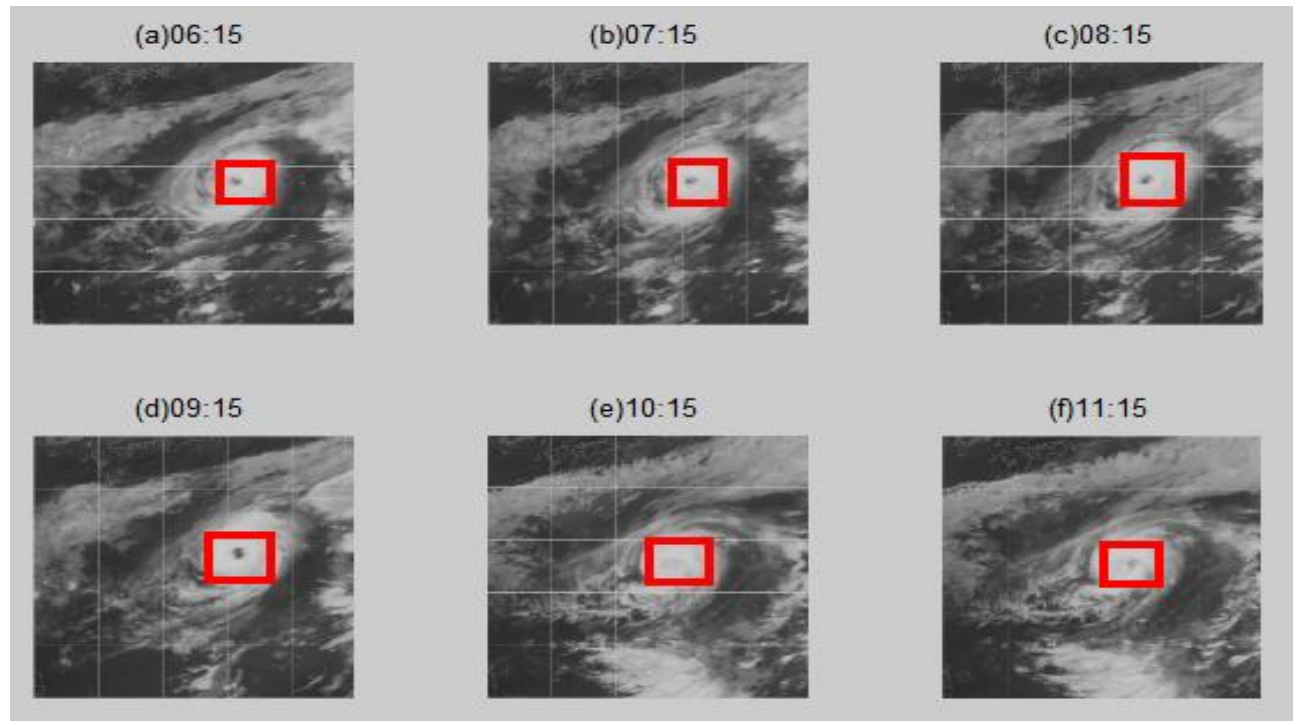

Figure 2. The Cloud Image in the Different Times of a Day

The Figure 2 shows the location of the cloud cluster. The cloud positions are obtained in the different times of a day. The cloud cluster is labeled by red rectangle, where the red rectangle represents a specific size and position of the cloud. It is once again proved that the cloud is constantly changing and moving. The Figure 3 illustrates the cloud cluster's moving trajectory. The size of the red rectangle indicates the size of the cloud cluster. The location of red rectangle represents the cloud cluster's position. This group of red rectangle indicates a right-down movement of the cloud cluster. The outmost red rectangle represents the future trend of the cloud cluster. This predicted value is calculated by using the curve fitting algorithm. And corrected value is achieved by using the motion vector. 


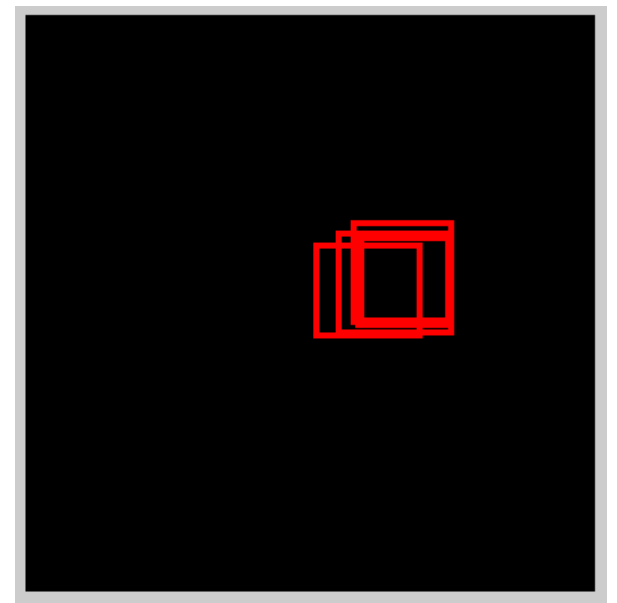

Figure 3. The Cloud Cluster's Moving Trajectory

There are the original image, the experiment predicted value and comparison image in the Figure 4. As shown in Figure 4, this depicts three images, the original image on the left-hand, the forecast image on the middle-hand and the comparison image on the right-hand. The results show, this method can predict the position of the cloud cluster well.

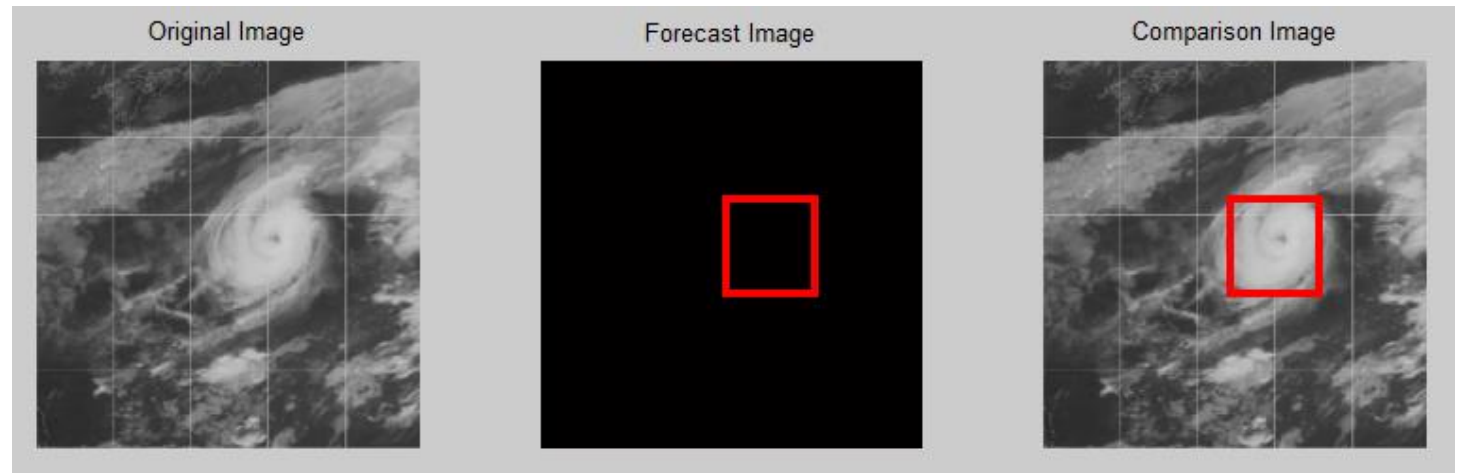

Figure 4. Experimental Results (1)

The Figure 4 depicts one hour forecast result. Here is look at two hour forecast, three hour forecast and so on. The results of the experiments can be seen in Figure 5. The cloud cluster moves to southwest. The time is shorter, and the result is closer to the actual. With the forecast time is longer, the error is bigger. 


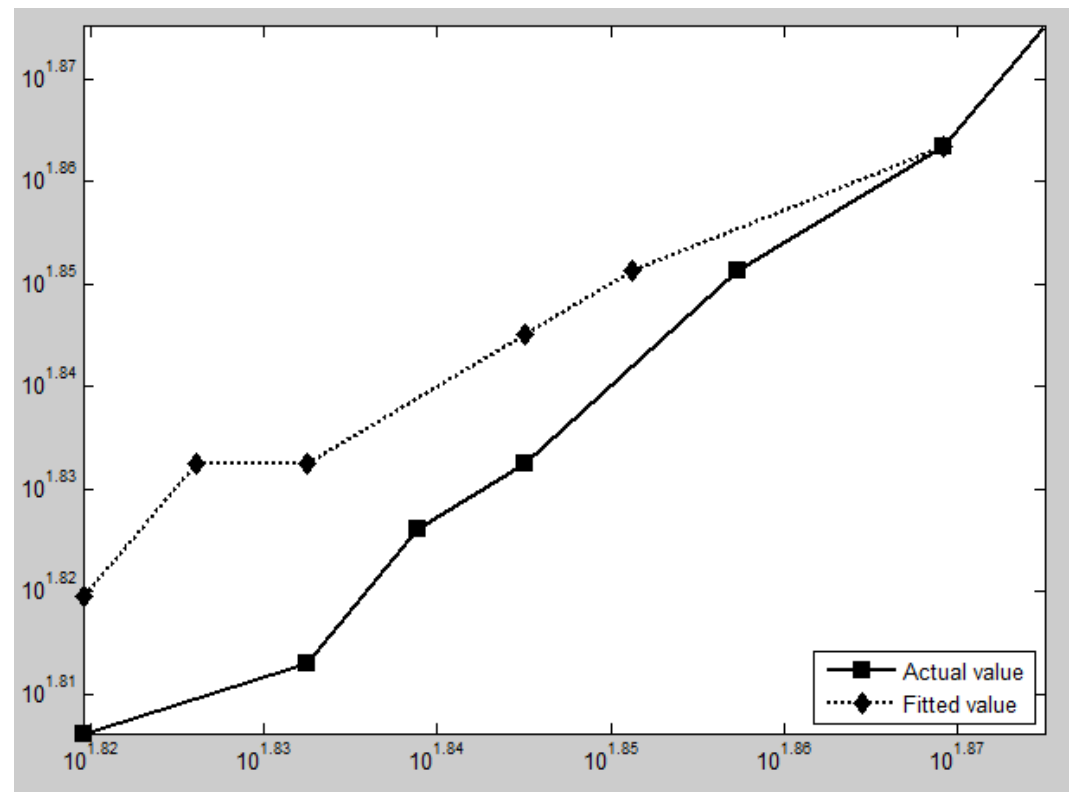

Figure 5. Experimental Results (2)

Through the analysis of experimental data, we can draw two conclusions. First of all, the algorithm can get good results for short time prediction. In particular, the forecast image which by one hour prediction or two hours prediction can accurately express the position of the cloud cluster. It is similar to the position of the original image. With the increase of time, the position will appear larger deviation. Secondly, the algorithm can get good results for the cloud cluster changes smoothly. Although the algorithm for cloud cluster changes unsmooth has improved, we still need to be optimized.

\section{Conclusions}

In this paper, we study methods of the short term prediction of satellite cloud image based on curve fitting and motion vector. We have discussed the meaning of reach the satellite cloud image. We review the sequential similarity detection algorithm, curve fitting and motion vector, and put forward a cloud cluster trajectory prediction based on curve fitting. The results show that this method has good prediction effect, and has a certain practical significance to the analysis and forecast of weather.

In cloud cluster trajectory prediction field, there are not any method could deal with this problem once and achieve best result. Sometimes we need use some method synthetically to achieve better result, sometimes need import new method. Although this paper puts forward a new research angle of view, the specific study is insufficient. We need to continue to study this further.

\section{References}

[1] D. E. Wolf, D. J. Hall and R. M. Endlich, "Experiments In Automatic Cloud Tracking Using SMS-GOES Data", Journal of Applied Meteorology, vol. 16, no. 11, (2010), pp. 1219-1230.

[2] L. Xuan, J. C. Ramella-Roman and H. Yong, "Robust spectral-domain optical coherence tomography speckle model and its cross-correlation coefficient analysis", Journal of the Optical Society of America an Optics Image Science \& Vision, vol. 30, no. 1, (2013), pp. 51-59.

[3] K. F. Liu, R. Zhang and Nanjing, "A Cloud Movement Short-time Forecast Based on Cross-correlation", Journal of Image \& Graphics, vol. 11, no. 4, (2006), pp. 586-591.

[4] K. F. Liu, Z. Ren and W.-C. Li, "Cloud cluster movement forecast technique of satellite cloud pictures based on singular value decomposition and artificial neural networks", Journal of Pla University of Science \& Technology, vol. 9, no. 3, (2008), pp. 298-301.

[5] J. G. Wang, R. Zhang and W.-X. Yu, "Non-linear Forecast Model of Cloud Clusters Movement Based on Parameters Retrieval of Historical Satellite Cloud Pictures Time Series", Journal of National University 
of Defense Technology, vol. 29, no. 5, (2007), pp. 41-47.

[6] W. Qiu, Z. Jian and L. Jie, "Image matching combine SIFT with regional SSDA", Control Engineering and Communication Technology (ICCECT), International Conference on, IEEE, (2012).

[7] X. Yu, X. Chen and M. Jiang, "Motion detection in moving background using a novel algorithm based on image features guiding self-adaptive Sequential Similarity Detection Algorithm", Optic-International Journal for Light and Electron Optics, vol. 123, no. 22, (2012), pp. 2031-2037.

[8] M. Talaat, "Short-Term Load Forecasting Using Curve Fitting Prediction Optimized by Genetic Algorithms", International Journal of Energy Engineering, vol. 7, no. 6, (2012), pp. 23-28.

[9] Y. Yao, W. Zhang and N. Yu, "Defining Embedding Distortion for Motion Vector-based Video Steganography", Multimedia Tools \& Applications, vol. 74, no. 24, (2014), pp. 1-24.

[10] H. Zhao, H. Shang and G. Jia, "Simulation of remote sensing imaging motion blur based on image motion vector field”, Journal of Applied Remote Sensing, vol. 8, no. 1, (2014), pp. 083539-083539.

\section{Authors}

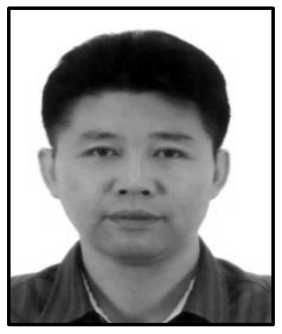

Huang Min (1972 ), he is a professor of zhengzhou university of light industry. The research fields are information process, image process. He has published more than 20 research papers on domestic or foreign well-known journals, more than 10 papers have been indexed by SCI/EI.

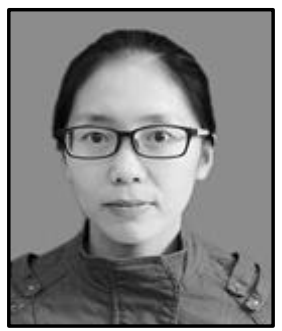

Wang Jiali, she received her bachelor's degree from zhengzhou university in 2013. She is currently a Master Degree from Zhengzhou University of Light Industry. Her research interests include signal and information processing. 